\title{
Molecular Dynamics of Dimethyldioxirane C-H Oxidation
}

\author{
Zhongyue Yang, Peiyuan Yu, K. N. Houk* \\ Department of Chemistry and Biochemistry, University of California, Los Angeles, California \\ 90095, United States
}

\section{Contents}

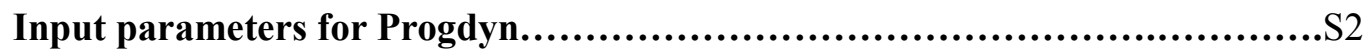

Transition state normal mode sampling..................................... 5

Charge distribution Analysis....................................................S6

Cartesian coordinates and energies...............................................S7 


\section{Input parameters for Progdyn}

The configuration file progdyn.conf for trajectory initialization with implicit acetone solvation is attached. For the gas phase MD simulation, the line specifying the solvation model, method $3 \operatorname{scrf}=($ smd,Solvent=acetone $)$, was commented.

method UB3LYP/6-31G*

method2 unrestricted

charge 0

multiplicity 1

processors 8

memory $200 \mathrm{mw}$

killcheck 0

diagnostics 0

title cf 2 cis 2 butene 298 dis 2

initialdis 2

timestep 1E-15

scaling 1.0

temperature 298.15

\#*** method3, method4, method5, and method6 -- These keywords let you add extra lines to the gaussian input file.

\#method 3 and method 4 add lines at the top of the input after the lines defining the method, and \#this is useful to implement things like the iop for mPW1k

\#method5 and method6 add lines after the geometry, after a blank line of course

\#only a single term with no spaces can be added, one per method line. Here are some examples to uncomment if needed

\#method3 IOp(3/76=0572004280)

method3 scrf=(smd,Solvent=acetone) [This line is commented in gas-phase MD simulations.]

\#add the line below with big structures to get it to put out the distance matrix and the input orientation

\#method3 iop(2/9=2000)

\#method4 scrf $=(\mathrm{pcm}$,solvent $=\mathrm{dmso}$,read $)$

\#method5 radii=bondi

\#method6

\#*** methodfile -- This keyword lets you add more complicated endings to gaussian input files \#such as a gen basis set. Put after the keyword the number of lines in a file you create called \#methodfile that contains the test you want to add to the end of the gaussian input methodfile 0

\#*** numimag --This tells the program the number of imaginary frequencies in the starting structure.

\#if 0 , treats as ground state and direction of all modes is random 
\#if 1, motion along the reaction coordinate will start out in the direction defined by searchdir

\#if 2, only lowest freq will go direction of searchdir and other imag mode will go in random direction

numimag 1

\#*** searchdir -- This keyword says what direction to follow the mode associated with the imaginary frequency.

\#The choices are "negative" and "positive". Positive moves in the direction defined in the gaussian frequency calculation

\#for the imaginary frequency, while negative moves in the opposite direction. The correct choice can be made either

\#by a careful inspection of the normal modes and standard orientation geometry, or by trial and error.

searchdir positive

\#*** classical -- for quassiclassical dynamics, the default, use 0 . for classical dynamics, use 1

\#if there are no normal modes and the velocities are to be generated from scratch, use classical 2

classical 0

\#*** DRP, saddlepoint, and maxAtomMove --to run a DRP use 'DRP 1 ' in the line below, otherwise leave it at 0 or comment it out

\#the treatment of starting saddlepoints is not yet implemented so use saddlepoint no

\#if DRP shows oscillations then decrease maxAtomMove

\#DRP 1

\#saddlepoint no

\#maxAtomMove 0.01

\#*** cannonball -- The program can "fire" a trajectory from a starting position toward a particular target, such as toward

\#a ts. To use this, make a file cannontraj with numAtom lines and three numbers per line that defines the vector

\#for firing the trajectory, relative to the starting geometry's standard orientation. The number following cannonball sets

\#the extra energy being put into the structure in $\mathrm{kcal} / \mathrm{mol}$

\#cannonball 10

\#*** keepevery --This tells the program how often to write the gaussian output file to file dyn, after the first two points.

\#Use 1 for most dynamics to start with, but use a higher number to save on disk space or molden loading time.

keepevery 1

\#*** highlevel --For ONIOM jobs, the following line states the number of highlevel atoms, \#which must come before the medium level atoms. Use some high value such as 999 if not using ONIOM

highlevel 999

\#*** fixedatom1, fixedatom2, fixedatom3, and fixedatom4 - These fix atoms in space.

\#Fixing one atom serves no useful purpose and messes things up, while fixing two atoms

\#fixes one distance and fixing three has the effect of fixing three distances, not just two \#in current form fixed atoms only are meant to work with no displacements, that is, initialdis $=0$ 
\#fixedatom1 2

\#fixedatom 23

\#fixedatom3 19

\#*** boxon and boxsize - With boxon 1, a cubic box is set such that atoms that reach the edge

\#are reflected back toward the middle. Useful for dynamics with solvent molecules. This is a crude

\#implementation that is ok for a few thousand femtoseconds but will not conserve energy long term.

\#Set the box size so as to fit the entire initial molecule but not have too much extra room.

\#The dimensions of the box are two times the boxsize, e.g. boxsize 7.5 leads to a box that is $15 \mathrm{x}$ $15 \times 15$ angstroms

boxon 0

boxsize 7.5

\#*** displacements -- This keyword lets you set the initialdis of particular modes by using a series of lines of the format

\# displacements NumberOfMode InitialDisForThatMode, as in the example below. You should be able to do as many of these as you like

\# you might consider this for rotations where a straight-line displacement goes wrong at large displacements

\# The choices for InitialDisForThatMode are $0,1,2$, and 10, where 10 does the same thing as 0 but is maintained for now because

\# a previous version of the program had a bug that made 0 not work.

\#displacements 10

\#*** etolerance --This sets the allowable difference between the desired energy in a trajectory and the actual

\#energy, known after point 1 from the potential energy + the kinetic energy in the initial velocities. \#The unit is $\mathrm{kcal} / \mathrm{mol}$ and 1 is a normal value for mid-sized organic systems. For very large and floppy molecules, a larger value

\#may be needed, but the value must stay way below the average thermal energy in the molecule (not counting zpe).

\#If initialdis is not 0 and few trajectories are being rejected, decrease the value.

etolerance 1

\#*** controlphase --It is sometimes useful to set the phase of particular modes in the initialization of trajectories.

\#The format is controlphase numberOfModeToControl positive or controlphase numberOfModeToControl negative.

\#controlphase 2 positive

\#*** damping -- The damping keyword lets you add or subtract energy from the system at each point, by multiplying the velocities

\#by the damping factor. A damping of 1 has no effect, and since you mostly want to change the energy slowly, normal values range

\#from 0.95 to 1.05 . The use of damping lets one do simulated annealing - you add energy until the structure is moving enough

\#to sample the kinds of possibilities you are interested in, then you take away the energy slowly. 
damping 1

\#*** reversetraj --This keyword sets the trajectories so that both directions from a transition state are explored.

reversetraj true

\#updated Aug 9, 2007 to include the possibility of classical dynamics by the keyword classical

\#updated Jan 2008 to include fixed atoms, ONIOM jobs, keepevery, and box size

\#update Feb 2008 to include methodfile parameter

\# updated Nov 2008 to allow for start without an initial freq calc using classical $=2$

\# update Aug 2010 to include etolerance, damping controlphase and reversetraj

\section{Transition state normal mode sampling}

Figure $\mathrm{S} 1$ shows the distribution of $\mathrm{O}_{\mathrm{D}}-\mathrm{H}_{B}$ and $\mathrm{O}_{\mathrm{D}}-\mathrm{C}_{\mathrm{B}}$ bond lengths from $\mathrm{TS}$ normal mode sampling at $300 \mathrm{~K}$. The distribution conforms to a Gaussian shape. The transition zones (98\% confidence interval of the Gaussian distributions) in the gas phase are $1.23 \pm 0.14 \AA$ for the $\mathrm{O}_{\mathrm{D}}-\mathrm{H}_{\mathrm{B}}$ bond and $2.50 \pm 0.12 \AA$ for the $\mathrm{O}_{\mathrm{D}}-\mathrm{C}_{\mathrm{B}}$ bond. In implicit acetone, these are $1.25 \pm 0.18 \AA$ for the $\mathrm{O}_{\mathrm{D}}-\mathrm{H}_{\mathrm{B}}$ bond and $2.50 \pm 0.12 \AA$ for the $\mathrm{O}_{\mathrm{D}}-\mathrm{C}_{\mathrm{B}}$ bond. The transition zones sampled in both cases are very similar, indicating that acetone solvation has little impact on the TS-1.

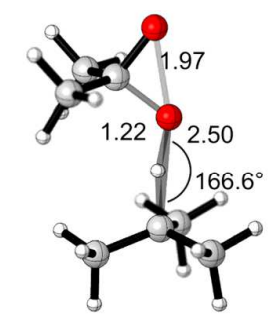

Gas phase

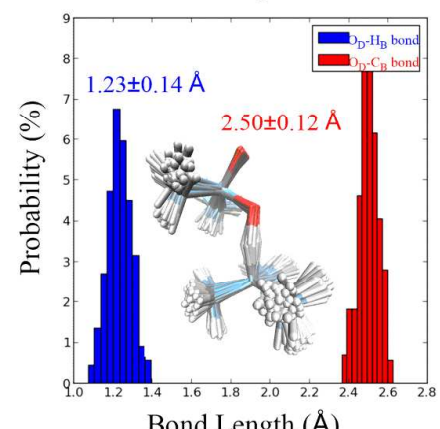

Bond Length $(\AA)$
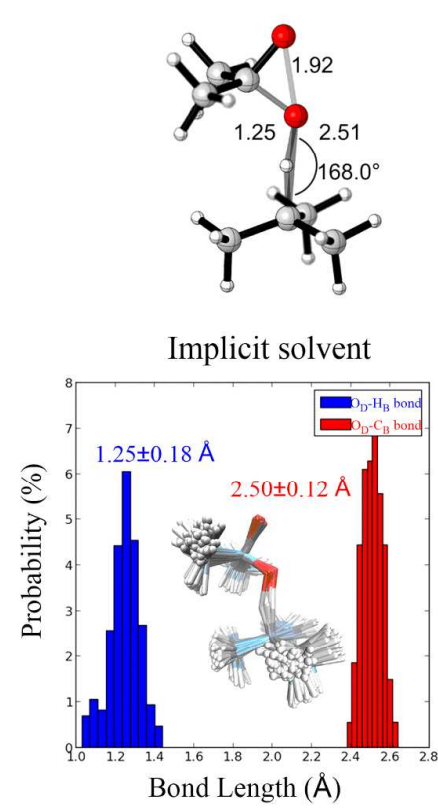

Figure S1. Distribution of $\mathrm{O}_{D}-\mathrm{H}_{B}$ and $\mathrm{O}_{\mathrm{D}}-\mathrm{C}_{\mathrm{B}}$ bond lengths from TS normal mode sampling at 
$300 \mathrm{~K}$ in the gas phase and in acetone solvation, respectively. Transition zones of $\mathrm{O}_{\mathrm{D}}-\mathrm{H}_{\mathrm{B}}$ bond and $\mathrm{O}_{\mathrm{D}}-\mathrm{C}_{\mathrm{B}}$ bond are labeled in blue and red, respectively.

\section{Charge distribution analysis on O-rebound TS-2}

The oxygen-rebound transition state TS-2 located in the gas phase cannot be located with solvation. Unrestricted single-point calculation with implicit solvent on gas phase optimized TS-2 geometry results in a substantially lower energy than that of Intermediate (Figure 2). We computed its charge distribution and dipole moment in the gas phase and in implicit acetone. The results are shown in Figure S2. With solvent, its singlet diradical character disappears and there is a substantial negative charge buildup on the terminal oxygen. The computed dipole moment also increases dramatically (from 6.0 Debye to 17 Debye).

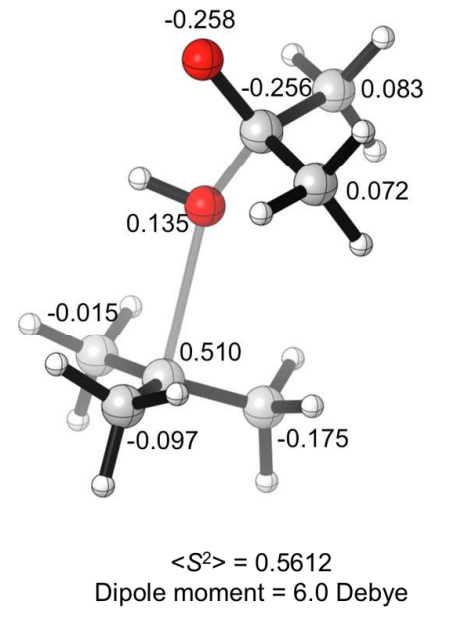

Gas phase UB3LYP/6-311++G(d,p)

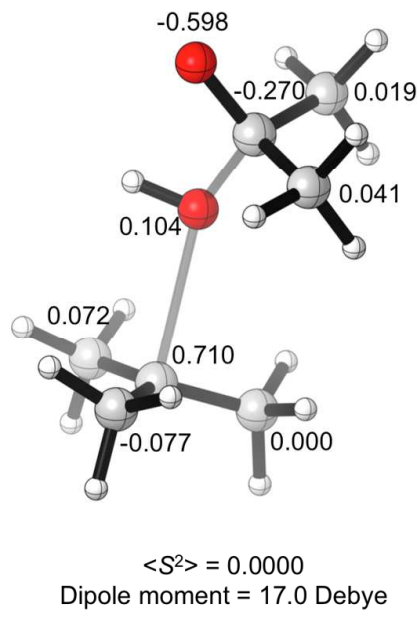

SMD(acetone)-UB3LYP/6-311++G(d,p)

Figure S2. Computed charges, $\left\langle S^{2}\right\rangle$ values and dipole moments on the O-rebound transition state TS-2. The two structures shown are the same one optimized in the gas phase at UB3LYP/6-31G(d) level of theory. Single point calculations in the gas phase (left) are compared to that with implicit acetone solvent (right). 


\section{Cartesian coordinates and energies}

Gas phase (U)B3LYP/6-31G(d) optimized structures

\section{DMDO}

$\begin{array}{lrrr}\mathrm{C} & 0.09412 & 0.00000 & 0.00000 \\ \mathrm{C} & 0.87206 & -1.29391 & 0.00000 \\ \mathrm{H} & 1.51387 & -1.35360 & -0.88614 \\ \mathrm{H} & 0.17838 & -2.13712 & 0.00004 \\ \mathrm{H} & 1.51393 & -1.35356 & 0.88610 \\ \mathrm{C} & 0.87206 & 1.29391 & -0.00000 \\ \mathrm{H} & 1.51387 & 1.35360 & 0.88614 \\ \mathrm{H} & 0.17838 & 2.13712 & -0.00004 \\ \mathrm{H} & 1.51393 & 1.35356 & -0.88610 \\ \mathrm{O} & -1.09012 & -0.00001 & -0.75276 \\ \mathrm{O} & -1.09012 & 0.00001 & 0.75276\end{array}$

\begin{tabular}{lccc}
\multicolumn{4}{l}{ Intermediate } \\
$\mathrm{C}$ & -2.19424 & 0.00337 & 0.01177 \\
$\mathrm{H}$ & 0.00889 & -0.10989 & -0.38159 \\
$\mathrm{C}$ & -2.21069 & 1.38339 & -0.57957 \\
$\mathrm{H}$ & -1.88025 & 2.14169 & 0.14034 \\
$\mathrm{H}$ & -1.57052 & 1.45057 & -1.46725 \\
$\mathrm{H}$ & -3.22957 & 1.67127 & -0.89856 \\
$\mathrm{C}$ & -2.37807 & -1.16096 & -0.91838 \\
$\mathrm{H}$ & -3.42992 & -1.24640 & -1.24907 \\
$\mathrm{H}$ & -1.76927 & -1.05789 & -1.82425 \\
$\mathrm{H}$ & -2.12109 & -2.11299 & -0.43872 \\
$\mathrm{C}$ & -2.58020 & -0.16538 & 1.45135 \\
$\mathrm{H}$ & -2.26605 & -1.13856 & 1.84860 \\
$\mathrm{H}$ & -2.15491 & 0.62217 & 2.08580 \\
$\mathrm{H}$ & -3.67719 & -0.11415 & 1.58446 \\
$\mathrm{O}$ & 0.81273 & -0.09910 & -0.93602 \\
$\mathrm{C}$ & 1.95532 & -0.01659 & -0.09264 \\
$\mathrm{O}$ & 3.08006 & -0.00311 & -0.82432 \\
$\mathrm{C}$ & 2.01864 & -1.25959 & 0.84518 \\
$\mathrm{C}$ & 1.91779 & 1.30377 & 0.73462 \\
$\mathrm{H}$ & 2.06657 & -2.16450 & 0.23475 \\
$\mathrm{H}$ & 2.89575 & -1.20889 & 1.49448 \\
$\mathrm{H}$ & 1.11283 & -1.29281 & 1.46040 \\
$\mathrm{H}$ & 2.79592 & 1.37919 & 1.38011 \\
$\mathrm{H}$ & 1.89313 & 2.15374 & 0.04857 \\
$\mathrm{H}$ & 1.01203 & 1.31701 & 1.35061
\end{tabular}




\begin{tabular}{|c|c|c|c|}
\hline \multicolumn{4}{|c|}{ tert-Butyl alcohol (TBA) } \\
\hline $\mathrm{C}$ & -0.00551 & 0.00000 & 0.01410 \\
\hline $\mathrm{C}$ & -1.49051 & -0.00002 & -0.35668 \\
\hline $\mathrm{H}$ & -1.98371 & 0.88647 & 0.05534 \\
\hline $\mathrm{H}$ & -1.98369 & -0.88652 & 0.05535 \\
\hline $\mathrm{H}$ & -1.62264 & -0.00002 & -1.44398 \\
\hline $\mathrm{C}$ & 0.69040 & 1.26559 & -0.51008 \\
\hline $\mathrm{H}$ & 0.65366 & 1.32109 & -1.60431 \\
\hline $\mathrm{H}$ & 1.74770 & 1.27952 & -0.21327 \\
\hline $\mathrm{H}$ & 0.20977 & 2.15976 & -0.09966 \\
\hline $\mathrm{O}$ & 0.01422 & 0.00000 & 1.45207 \\
\hline $\mathrm{H}$ & 0.94506 & 0.00001 & 1.72759 \\
\hline $\mathrm{C}$ & 0.69043 & -1.26557 & -0.51008 \\
\hline $\mathrm{H}$ & 0.65369 & -1.32108 & -1.60431 \\
\hline $\mathrm{H}$ & 0.20982 & -2.15975 & -0.09966 \\
\hline $\mathrm{H}$ & 1.74774 & -1.27948 & -0.21327 \\
\hline
\end{tabular}

\section{TS-1}

$\begin{array}{lrrr}\mathrm{C} & 1.75168 & -0.00632 & 0.01550 \\ \mathrm{C} & 1.79710 & -0.59617 & 1.41193 \\ \mathrm{C} & 2.37933 & -0.87040 & -1.06188 \\ \mathrm{C} & 2.09516 & 1.46870 & -0.07122 \\ \mathrm{H} & 0.50607 & -0.02809 & -0.32755 \\ \mathrm{H} & 2.83880 & -0.62159 & 1.76758 \\ \mathrm{H} & 1.41930 & -1.62366 & 1.43055 \\ \mathrm{H} & 1.22385 & 0.00242 & 2.12831 \\ \mathrm{H} & 3.46939 & -0.91705 & -0.91573 \\ \mathrm{H} & 2.19148 & -0.46122 & -2.05931 \\ \mathrm{H} & 1.99324 & -1.89416 & -1.03054 \\ \mathrm{H} & 3.16471 & 1.61537 & 0.14370 \\ \mathrm{H} & 1.52737 & 2.06073 & 0.65418 \\ \mathrm{H} & 1.89696 & 1.86383 & -1.07223 \\ \mathrm{O} & -0.56581 & -0.01774 & -0.91520 \\ \mathrm{O} & -2.51272 & 0.05896 & -1.18986 \\ \mathrm{C} & -1.71895 & 0.00151 & -0.07079 \\ \mathrm{C} & -1.79501 & 1.26456 & 0.78864 \\ \mathrm{C} & -1.89307 & -1.29628 & 0.71924 \\ \mathrm{H} & -1.64115 & 2.13959 & 0.15200 \\ \mathrm{H} & -1.04212 & 1.25745 & 1.58648 \\ & & & \\ \mathrm{H} & -2.78165 & 1.34092 & 1.25957 \\ \mathrm{H} & -1.78967 & -2.14458 & 0.03780 \\ \mathrm{H} & -2.89074 & -1.32882 & 1.17191\end{array}$


TS-2

$\mathrm{C}$

$$
2.06099
$$

$-0.00809$

0.04434

C

$$
2.46398
$$

1.22915

$-0.68555$

C

0.05645

1.52856

C

1.93866

$-1.33574$

$-0.62370$

$\mathrm{H}$

2.17730

0.60144

$-1.29501$

1.46399

$-0.50523$

$\mathrm{H}$

3.52894

2.09567

$-0.34175$

1.12910

$-1.76932$

0.06913

2.00407

2.93628

$-0.80739$

1.92998

1.42226

0.96966

1.84378

$\mathrm{H} \quad 3.20217$

$-1.73859$

$-0.52044$

1.96885

$-1.27423$

$-1.69706$

$-2.07094$

$-0.17573$

1.50097

0.40323

$-0.34276$

0.00081

$-1.30416$

$-2.45089$

$-0.0113$

$-0.14805$

1.06750

0.79512

$-1.39993$

0.52569

2.03137

0.28077

1.13942

1.70113

0.79928

1.06241

$-2.12335$

$-0.16164$

$-1.71830$

0.74460

$-1.38684$

1.45634

\section{Acetone}

C

C

$\mathrm{H}$

$\mathrm{H}$

$\mathrm{H}$

C

$\mathrm{H}$

$\mathrm{H}$

$\mathrm{H}$

$\mathrm{O}$

\section{Isobutane}

C

C

0.18537
-0.61483
-1.26694
0.06343
-1.26772
-0.61483
-1.26771
0.06343
-1.26696
1.40103

$-0.00004$

$-0.00001$

0.88127

$-0.00042$

$-0.88072$

$-0.00001$

$-0.88073$

$-0.00040$

0.88126

0.00001

$$
-0.00015
$$

1.45892

$$
\begin{array}{r}
-0.37308 \\
0.09579
\end{array}
$$




\begin{tabular}{|c|c|c|c|}
\hline $\mathrm{C}$ & -1.30942 & -0.65026 & 0.09592 \\
\hline $\mathrm{C}$ & 1.21798 & -0.80860 & 0.09585 \\
\hline $\mathrm{H}$ & -0.00016 & -0.00017 & -1.47362 \\
\hline $\mathrm{H}$ & 0.09717 & 1.51699 & 1.19238 \\
\hline $\mathrm{H}$ & -0.76047 & 2.04874 & -0.26341 \\
\hline $\mathrm{H}$ & 1.00859 & 1.93858 & -0.26671 \\
\hline $\mathrm{H}$ & -1.36206 & -0.67538 & 1.19249 \\
\hline $\mathrm{H}$ & -1.39478 & -1.68250 & -0.26438 \\
\hline $\mathrm{H}$ & -2.18318 & -0.09501 & -0.26586 \\
\hline $\mathrm{H}$ & 1.26745 & -0.83968 & 1.19242 \\
\hline $\mathrm{H}$ & 2.15446 & -0.36743 & -0.26616 \\
\hline $\mathrm{H}$ & 1.17325 & -1.84355 & -0.26404 \\
\hline \multicolumn{4}{|l|}{1} \\
\hline $\mathrm{C}$ & 1.85743 & -0.59704 & 0.17311 \\
\hline $\mathrm{C}$ & 0.74413 & -0.19740 & -0.78524 \\
\hline $\mathrm{C}$ & 1.42932 & -1.54933 & -0.90509 \\
\hline $\mathrm{H}$ & 1.03479 & 0.53167 & -1.54001 \\
\hline $\mathrm{C}$ & -0.67435 & -0.06187 & -0.34646 \\
\hline $\mathrm{H}$ & 2.09414 & -1.70011 & -1.75282 \\
\hline $\mathrm{H}$ & 0.85465 & -2.43028 & -0.63053 \\
\hline $\mathrm{H}$ & 1.52137 & -0.84026 & 1.18150 \\
\hline $\mathrm{C}$ & 3.20274 & 0.10187 & 0.11586 \\
\hline $\mathrm{C}$ & -1.46772 & 0.96609 & -0.87760 \\
\hline $\mathrm{C}$ & -1.26083 & -0.92627 & 0.59230 \\
\hline $\mathrm{C}$ & -2.58901 & -0.76597 & 0.98465 \\
\hline $\mathrm{C}$ & -3.36560 & 0.26263 & 0.44708 \\
\hline $\mathrm{C}$ & -2.79727 & 1.12865 & -0.48758 \\
\hline $\mathrm{H}$ & -1.03480 & 1.64631 & -1.60793 \\
\hline $\mathrm{H}$ & -3.38870 & 1.93380 & -0.91631 \\
\hline $\mathrm{H}$ & -3.01894 & -1.44870 & 1.71333 \\
\hline $\mathrm{H}$ & -4.40098 & 0.38612 & 0.75287 \\
\hline $\mathrm{H}$ & -0.67671 & -1.73552 & 1.02387 \\
\hline $\mathrm{H}$ & 3.97786 & -0.57616 & 0.50096 \\
\hline $\mathrm{C}$ & 3.23172 & 1.41730 & 0.90323 \\
\hline $\mathrm{H}$ & 3.46257 & 0.29398 & -0.93461 \\
\hline $\mathrm{H}$ & 3.00491 & 1.24823 & 1.96284 \\
\hline $\mathrm{H}$ & 2.48963 & 2.12492 & 0.51602 \\
\hline $\mathrm{H}$ & 4.21691 & 1.89400 & 0.84533 \\
\hline
\end{tabular}

$\begin{array}{lrcc}\text { Int-a } & & & \\ \mathrm{C} & -0.70406 & 1.72548 & 0.13026 \\ \mathrm{H} & -2.35716 & 0.32569 & -0.38073 \\ \mathrm{C} & 0.16828 & 0.55810 & 0.19603\end{array}$




$\begin{array}{lrrc}\mathrm{C} & 0.38093 & -0.21768 & 1.47179 \\ \mathrm{H} & 0.25452 & -0.01409 & -0.72643 \\ \mathrm{C} & 1.52698 & 0.62506 & 0.99130 \\ \mathrm{C} & -0.91442 & 2.47795 & -1.14723 \\ \mathrm{H} & -0.05226 & 3.12590 & -1.38447 \\ \mathrm{H} & -1.04590 & 1.79948 & -1.99872 \\ \mathrm{H} & -1.79506 & 3.12745 & -1.09331 \\ \mathrm{H} & -0.92627 & 2.23645 & 1.06759 \\ \mathrm{O} & -2.90970 & -0.26715 & -0.92580 \\ \mathrm{C} & -4.07331 & -0.61661 & -0.18820 \\ \mathrm{O} & -4.88001 & -1.39947 & -0.92147 \\ \mathrm{C} & -4.87399 & 0.66512 & 0.19088 \\ \mathrm{C} & -3.67704 & -1.42197 & 1.08691 \\ \mathrm{H} & -5.15652 & 1.19235 & -0.72351 \\ \mathrm{H} & -5.77214 & 0.40604 & 0.75636 \\ \mathrm{H} & -4.23863 & 1.31996 & 0.79720 \\ \mathrm{H} & -4.56455 & -1.69817 & 1.66100 \\ \mathrm{H} & -3.14040 & -2.32448 & 0.78494 \\ \mathrm{H} & -3.01657 & -0.80741 & 1.70806 \\ \mathrm{H} & 1.67201 & 1.56178 & 1.52413 \\ \mathrm{C} & 2.77068 & 0.06349 & 0.40375 \\ \mathrm{H} & 0.47390 & -1.29820 & 1.40075 \\ \mathrm{H} & -0.14787 & 0.12253 & 2.35981 \\ \mathrm{C} & 2.81399 & -1.19261 & -0.22428 \\ \mathrm{C} & 3.95567 & 0.81463 & 0.45949 \\ \mathrm{C} & 5.14297 & 0.33005 & -0.08779 \\ \mathrm{C} & 5.17089 & -0.91922 & -0.70926 \\ \mathrm{C} & 3.99944 & -1.67676 & -0.77495 \\ \mathrm{H} & 6.04701 & 0.93059 & -0.02794 \\ \mathrm{H} & 6.09435 & -1.29930 & -1.13735 \\ \mathrm{H} & 3.94379 & 1.78971 & 0.94150 \\ \mathrm{H} & 1.91577 & -1.80200 & -0.28711 \\ \mathrm{H} & 4.00761 & -2.65155 & -1.25575\end{array}$

\section{TS-1a}

$\begin{array}{lrrr}\mathrm{C} & -0.96969 & 0.92660 & 0.01293 \\ \mathrm{H} & -1.22264 & 1.12465 & 1.06147 \\ \mathrm{C} & 0.14449 & -0.03721 & -0.14374 \\ \mathrm{C} & -0.94793 & 2.16956 & -0.85225 \\ \mathrm{H} & -2.01595 & 0.28563 & -0.37596 \\ \mathrm{C} & 1.33184 & 0.00348 & 0.86021 \\ \mathrm{H} & 0.44166 & -0.23077 & -1.17265 \\ \mathrm{C} & 0.36947 & -1.15506 & 0.83355 \\ \mathrm{H} & -0.11077 & 2.82021 & -0.56180\end{array}$




$\begin{array}{lrrr}\mathrm{H} & -1.87399 & 2.74282 & -0.74879 \\ \mathrm{H} & -0.82038 & 1.91232 & -1.90886 \\ \mathrm{O} & -2.98967 & -0.27127 & -0.87616 \\ \mathrm{O} & -4.81028 & -1.02719 & -0.99667 \\ \mathrm{C} & -4.13786 & -0.31026 & -0.03433 \\ \mathrm{C} & -4.73697 & 1.07737 & 0.20574 \\ \mathrm{C} & -3.90007 & -1.10870 & 1.24954 \\ \mathrm{H} & -4.85233 & 1.58604 & -0.75484 \\ \mathrm{H} & -4.10330 & 1.68393 & 0.86462 \\ \mathrm{H} & -5.72252 & 0.98826 & 0.67708 \\ \mathrm{H} & -3.44167 & -2.06714 & 0.99260 \\ \mathrm{H} & -4.85238 & -1.29798 & 1.75784 \\ \mathrm{H} & -3.24868 & -0.56911 & 1.94814 \\ \mathrm{H} & 1.19453 & 0.70166 & 1.68310 \\ \mathrm{C} & 2.73578 & -0.08243 & 0.37522 \\ \mathrm{H} & 0.72218 & -2.10595 & 0.44447 \\ \mathrm{H} & -0.32464 & -1.24710 & 1.66544 \\ \mathrm{C} & 3.70033 & 0.78427 & 0.91201 \\ \mathrm{C} & 3.14201 & -0.99652 & -0.61087 \\ \mathrm{C} & 4.46637 & -1.03995 & -1.04271 \\ \mathrm{C} & 5.41595 & -0.17254 & -0.49789 \\ \mathrm{C} & 5.02660 & 0.73987 & 0.48337 \\ \mathrm{H} & 3.40590 & 1.49880 & 1.67748 \\ \mathrm{H} & 2.42170 & -1.68391 & -1.04763 \\ \mathrm{H} & 4.75777 & -1.75612 & -1.80648 \\ \mathrm{H} & 6.44821 & -0.20913 & -0.83471 \\ \mathrm{H} & 5.75516 & 1.41961 & 0.91739\end{array}$

\section{TS-2a}

$\begin{array}{lrcc}\mathrm{C} & 0.77943 & 1.55349 & -0.08500 \\ \mathrm{C} & -0.10916 & 0.43019 & 0.03532 \\ \mathrm{C} & 1.14793 & 2.41884 & 1.06786 \\ \mathrm{H} & 3.50477 & 1.17958 & -0.87670 \\ \mathrm{C} & -1.35075 & 0.32995 & -0.95697 \\ \mathrm{H} & -0.33750 & 0.09707 & 1.04498 \\ \mathrm{C} & -0.20485 & -0.61652 & -1.05647 \\ \mathrm{H} & 0.39535 & 3.21266 & 1.21999 \\ \mathrm{H} & 2.10883 & 2.91560 & 0.90467 \\ \mathrm{H} & 1.20599 & 1.84729 & 2.00013 \\ \mathrm{O} & 2.96401 & 0.43533 & -0.55752 \\ \mathrm{O} & 5.19758 & 0.13855 & -0.03409 \\ \mathrm{C} & 4.01595 & -0.44304 & 0.05364 \\ \mathrm{C} & 3.99309 & -1.74045 & -0.80360 \\ \mathrm{C} & 3.55966 & -0.71562 & 1.50365\end{array}$




$\begin{array}{lrrr}\mathrm{H} & 4.30001 & -1.49874 & -1.82406 \\ \mathrm{H} & 2.98092 & -2.15794 & -0.81717 \\ \mathrm{H} & 4.68632 & -2.47525 & -0.38407 \\ \mathrm{H} & 3.58820 & 0.22043 & 2.06926 \\ \mathrm{H} & 4.24714 & -1.42540 & 1.97462 \\ \mathrm{H} & 2.54331 & -1.12304 & 1.53854 \\ \mathrm{H} & 0.99871 & 1.91152 & -1.08689 \\ \mathrm{H} & -1.36934 & 1.12934 & -1.69330 \\ \mathrm{C} & -2.68579 & -0.02507 & -0.41333 \\ \mathrm{H} & -0.35550 & -1.64387 & -0.73666 \\ \mathrm{H} & 0.48878 & -0.50417 & -1.88406 \\ \mathrm{C} & -3.78913 & 0.78363 & -0.73150 \\ \mathrm{C} & -2.89659 & -1.13363 & 0.42387 \\ \mathrm{C} & -4.16578 & -1.42027 & 0.92326 \\ \mathrm{C} & -5.05964 & 0.49505 & -0.23614 \\ \mathrm{C} & -5.25424 & -0.60868 & 0.59582 \\ \mathrm{H} & -5.89803 & 1.13387 & -0.50105 \\ \mathrm{H} & -6.24319 & -0.83569 & 0.98403 \\ \mathrm{H} & -3.64666 & 1.64607 & -1.37877 \\ \mathrm{H} & -2.06638 & -1.78330 & 0.68913 \\ \mathrm{H} & -4.30524 & -2.28411 & 1.56788\end{array}$

\section{TS-3a}

$\begin{array}{lrrc}\mathrm{C} & -0.70657 & 1.44920 & 0.11934 \\ \mathrm{H} & -2.52359 & 0.28770 & -0.59928 \\ \mathrm{C} & 0.15072 & 0.33212 & 0.08559 \\ \mathrm{C} & 0.43978 & -0.55731 & 1.25223 \\ \mathrm{H} & 0.47299 & -0.03197 & -0.88702 \\ \mathrm{C} & 1.62710 & 0.32735 & 1.11462 \\ \mathrm{C} & -0.88705 & 2.39036 & -1.03434 \\ \mathrm{H} & -0.39232 & 3.35745 & -0.85166 \\ \mathrm{H} & -0.46857 & 1.97526 & -1.95762 \\ \mathrm{H} & -1.94783 & 2.60456 & -1.21701 \\ \mathrm{H} & -1.10645 & 1.76035 & 1.08564 \\ \mathrm{O} & -3.35637 & 0.12771 & -1.08361 \\ \mathrm{C} & -4.30667 & -0.44068 & -0.19302 \\ \mathrm{O} & -5.47046 & -0.66315 & -0.82400 \\ \mathrm{C} & -4.59223 & 0.53751 & 0.98604 \\ \mathrm{C} & -3.79196 & -1.81403 & 0.33533 \\ \mathrm{H} & -4.97882 & 1.47706 & 0.58386 \\ \mathrm{H} & -5.32143 & 0.10761 & 1.67670 \\ \mathrm{H} & -3.65740 & 0.73797 & 1.52050 \\ \mathrm{H} & -4.51880 & -2.26310 & 1.01622 \\ \mathrm{H} & -3.62381 & -2.48119 & -0.51341\end{array}$




$\begin{array}{lrrr}\mathrm{H} & -2.84351 & -1.66330 & 0.86234 \\ \mathrm{H} & 1.63166 & 1.22238 & 1.72823 \\ \mathrm{C} & 2.89020 & -0.03106 & 0.48259 \\ \mathrm{H} & 0.57610 & -1.61764 & 1.04298 \\ \mathrm{H} & -0.17265 & -0.39106 & 2.13902 \\ \mathrm{C} & 3.08991 & -1.24617 & -0.20709 \\ \mathrm{C} & 3.97172 & 0.87448 & 0.54222 \\ \mathrm{C} & 5.19368 & 0.57856 & -0.05237 \\ \mathrm{C} & 5.37294 & -0.62948 & -0.73300 \\ \mathrm{C} & 4.31291 & -1.53686 & -0.80582 \\ \mathrm{H} & 6.01041 & 1.29274 & 0.01401 \\ \mathrm{H} & 6.32633 & -0.86085 & -1.19950 \\ \mathrm{H} & 3.84124 & 1.81748 & 1.06831 \\ \mathrm{H} & 2.28361 & -1.97137 & -0.27538 \\ \mathrm{H} & 4.44069 & -2.48051 & -1.33004\end{array}$

Energetics are given in hartrees.

$\mathrm{E}=$ Electronic energy

$\mathrm{H}=$ Enthalpy

$\mathrm{G}=$ Gibbs free energy

\section{(U)B3LYP/6-31G(d)}

DMDO $\quad-268.268936$

Intermediatı -426.712671

TBA $\quad-233.670958$

TS-1 $\quad-426.688706 \quad-426$

TS-2

Acetone

Isobutane

1

Int-a

TS-1a

TS-2a

TS-3a

\section{E}

$H$ $-268.1736$ $-426.479635$ $-233.52711$

$-426.460378$

$-426.479128$

$-193.065254$ $-193.155695$

$-158.458813$

$-427.580518$

$-695.834212$

$-695.810159$

$-695.831199$

$-695.830957$
$-158.319781$

$-427.349114$

$-695.508937$

$-695.489414$

$-695.507156$

$-695.506996$

\section{G}

$-268.208524$

$-426.538279$

$-233.563785$

$-426.512879$

$-426.535068$

$-193.100249$

$-158.354027$

$-427.396784$

$-695.580698$

$-695.556296$

$-695.5752$

$-695.577151$
(U)B3LYP/6-311++G(d,p) E

$-268.349572$

$-426.851543$

$-233.752361$

$-426.82422$

$-426.852604$

$-193.218183$

$-158.506451$

$-427.692198$

$-696.037733$

$-696.009691$

$-696.038131$

$-696.034903$ 
SMD(acetone)-(U)B3LYP/6-31G(d) optimized structures

$\begin{array}{lrrr}\text { DMDO } & & & \\ \mathrm{C} & 0.00000 & 0.00000 & 0.09653 \\ \mathrm{C} & 0.00000 & 1.29032 & 0.87407 \\ \mathrm{H} & -0.88625 & 1.34310 & 1.51628 \\ \mathrm{H} & 0.00003 & 2.14235 & 0.18968 \\ \mathrm{H} & 0.88622 & 1.34307 & 1.51633 \\ \mathrm{C} & 0.00000 & -1.29032 & 0.87407 \\ \mathrm{H} & 0.88625 & -1.34310 & 1.51628 \\ \mathrm{H} & -0.00003 & -2.14235 & 0.18968 \\ \mathrm{H} & -0.88622 & -1.34307 & 1.51633 \\ \mathrm{O} & -0.75169 & 0.00000 & -1.09454 \\ \mathrm{O} & 0.75169 & -0.00000 & -1.09454\end{array}$

\section{Intermediate}

$\begin{array}{lccc}\mathrm{C} & -2.17047 & 0.00442 & 0.01524 \\ \mathrm{H} & 0.01014 & 0.01374 & -0.46252 \\ \mathrm{C} & -2.34436 & 1.41915 & -0.45201 \\ \mathrm{H} & -1.87586 & 2.13748 & 0.23245 \\ \mathrm{H} & -1.93035 & 1.57579 & -1.45574 \\ \mathrm{H} & -3.41570 & 1.69105 & -0.50660 \\ \mathrm{C} & -2.44321 & -1.10223 & -0.96060 \\ \mathrm{H} & -3.53050 & -1.26976 & -1.08008 \\ \mathrm{H} & -2.04963 & -0.87633 & -1.95910 \\ \mathrm{H} & -2.01649 & -2.05694 & -0.62791 \\ \mathrm{C} & -2.35742 & -0.28315 & 1.47535 \\ \mathrm{H} & -1.96582 & -1.26941 & 1.75416 \\ \mathrm{H} & -1.87880 & 0.47374 & 2.10957 \\ \mathrm{H} & -3.43035 & -0.28430 & 1.74693 \\ \mathrm{O} & 0.83023 & 0.03388 & -0.99752 \\ \mathrm{C} & 1.95284 & -0.00977 & -0.12172 \\ \mathrm{O} & 3.09143 & -0.03040 & -0.82582 \\ \mathrm{C} & 1.90385 & -1.29789 & 0.75577 \\ \mathrm{C} & 1.97987 & 1.26211 & 0.78270 \\ \mathrm{H} & 1.91076 & -2.17969 & 0.10906 \\ \mathrm{H} & 2.76122 & -1.33366 & 1.43228 \\ \mathrm{H} & 0.97987 & -1.29242 & 1.34304 \\ \mathrm{H} & 2.83545 & 1.23302 & 1.46176 \\ \mathrm{H} & 2.04104 & 2.15455 & 0.15380 \\ \mathrm{H} & 1.05514 & 1.29953 & 1.36735\end{array}$

tert-Butyl alcohol (TBA)

$\begin{array}{llll}\mathrm{C} & 0.00677 & 0.00000 & 0.01396\end{array}$ 


$\begin{array}{lrrr}\mathrm{C} & 1.49813 & 0.00001 & -0.32802 \\ \mathrm{H} & 1.98845 & -0.88780 & 0.08802 \\ \mathrm{H} & 1.98844 & 0.88783 & 0.08802 \\ \mathrm{H} & 1.64941 & 0.00001 & -1.41288 \\ \mathrm{C} & -0.67875 & -1.26407 & -0.52357 \\ \mathrm{H} & -0.62636 & -1.31428 & -1.61768 \\ \mathrm{H} & -1.73932 & -1.28016 & -0.23933 \\ \mathrm{H} & -0.20259 & -2.16246 & -0.11402 \\ \mathrm{O} & -0.04796 & 0.00000 & 1.45584 \\ \mathrm{H} & -0.99026 & -0.00000 & 1.69944 \\ \mathrm{C} & -0.67876 & 1.26406 & -0.52358 \\ \mathrm{H} & -0.62638 & 1.31427 & -1.61768 \\ \mathrm{H} & -0.20262 & 2.16246 & -0.11403 \\ \mathrm{H} & -1.73934 & 1.28014 & -0.23933\end{array}$

TS-1

$\begin{array}{lrrr}\mathrm{C} & 1.75101 & -0.00539 & 0.01592 \\ \mathrm{C} & 1.75426 & -0.55845 & 1.42675 \\ \mathrm{C} & 2.40172 & -0.90139 & -1.01932 \\ \mathrm{C} & 2.10906 & 1.46313 & -0.09627 \\ \mathrm{H} & 0.52553 & -0.02686 & -0.33924 \\ \mathrm{H} & 2.78983 & -0.57520 & 1.80042 \\ \mathrm{H} & 1.37561 & -1.58516 & 1.46331 \\ \mathrm{H} & 1.16964 & 0.06354 & 2.11291 \\ \mathrm{H} & 3.48394 & -0.95307 & -0.82571 \\ \mathrm{H} & 2.26500 & -0.51282 & -2.03398 \\ \mathrm{H} & 2.00719 & -1.92209 & -0.97571 \\ \mathrm{H} & 3.16909 & 1.59810 & 0.16731 \\ \mathrm{H} & 1.51683 & 2.08005 & 0.58800 \\ \mathrm{H} & 1.97141 & 1.83588 & -1.11658 \\ \mathrm{O} & -0.57519 & -0.01144 & -0.92495 \\ \mathrm{O} & -2.47013 & 0.04319 & -1.21819 \\ \mathrm{C} & -1.72764 & 0.00119 & -0.07226 \\ \mathrm{C} & -1.81707 & 1.26436 & 0.78047 \\ \mathrm{C} & -1.89363 & -1.28986 & 0.72500 \\ \mathrm{H} & -1.66093 & 2.14487 & 0.14998 \\ \mathrm{H} & -1.07243 & 1.26011 & 1.58466 \\ \mathrm{H} & -2.80910 & 1.33550 & 1.24241 \\ \mathrm{H} & -1.76276 & -2.15000 & 0.06137 \\ \mathrm{H} & -2.90058 & -1.33205 & 1.15749 \\ \mathrm{H} & -1.17194 & -1.35629 & 1.54679\end{array}$

Acetone

$\begin{array}{llll}\text { C } & 0.00000 & 0.18285 & -0.00001\end{array}$ 


$\begin{array}{lrrr}\mathrm{C} & 1.28744 & -0.61462 & -0.00214 \\ \mathrm{H} & 1.38885 & -1.15273 & 0.94972 \\ \mathrm{H} & 2.14655 & 0.04776 & -0.13446 \\ \mathrm{H} & 1.27456 & -1.37201 & -0.79544 \\ \mathrm{C} & -1.28744 & -0.61462 & 0.00214 \\ \mathrm{H} & -1.38880 & -1.15286 & -0.94965 \\ \mathrm{H} & -2.14656 & 0.04778 & 0.13433 \\ \mathrm{H} & -1.27461 & -1.37190 & 0.79554 \\ \mathrm{O} & 0.00000 & 1.40404 & 0.00000\end{array}$

$\begin{array}{lccc}\text { Isobutane } & & & \\ \mathrm{C} & -0.00024 & 0.00028 & -0.37326 \\ \mathrm{C} & 1.09986 & 0.96211 & 0.09609 \\ \mathrm{C} & -1.38368 & 0.47086 & 0.09607 \\ \mathrm{C} & 0.28378 & -1.43306 & 0.09595 \\ \mathrm{H} & 0.00006 & 0.00035 & -1.47385 \\ \mathrm{H} & 1.14657 & 1.00038 & 1.19304 \\ \mathrm{H} & 0.92072 & 1.98296 & -0.26451 \\ \mathrm{H} & 2.08690 & 0.64879 & -0.26702 \\ \mathrm{H} & -1.44047 & 0.49116 & 1.19305 \\ \mathrm{H} & -2.17721 & -0.19562 & -0.26496 \\ \mathrm{H} & -1.60705 & 1.48230 & -0.26633 \\ \mathrm{H} & 0.29102 & -1.49309 & 1.19293 \\ \mathrm{H} & 1.25959 & -1.78537 & -0.26191 \\ \mathrm{H} & -0.47839 & -2.13294 & -0.26953\end{array}$

Energetics are given in hartrees.

$\mathrm{E}=$ Electronic energy

$\mathrm{H}=$ Enthalpy

$\mathrm{G}=$ Gibbs free energy

\begin{tabular}{lcccc}
\multicolumn{2}{l}{$\mathrm{SMD}$ (acetone)-(U)B3LYP/6-31G(d) } & & $(\mathrm{U}) \mathrm{B} 3 \mathrm{LYP} / 6-311++\mathrm{G}(\mathrm{d}, \mathrm{p})$ \\
& $E$ & $H$ & $G$ & $E$ \\
DMDO & -268.280359 & -268.185811 & -268.2204 & -268.36249 \\
Intermediatt & -426.726365 & -426.494105 & -426.551628 & -426.868234 \\
TBA & -233.678537 & -233.535228 & -233.571951 & -233.761201 \\
TS-1 & -426.704644 & -426.476403 & -426.52842 & -426.843251 \\
Acetone & -193.16448 & -193.074185 & -193.107856 & -193.228964 \\
Isobutane & -158.46186 & -158.323448 & -158.357736 & -158.509841
\end{tabular}

